\title{
Structural Requirement of Fibrogenic Peptide AG97 (SAKVDAIGLEIV) and B160 (VILQQSAADIAR) for Amyloid-Like Fibril Formation and Cellular Activity
}

\author{
Fumihiko Katagiri, Kazuki Takeyama, Nobuko Yamada, Atsushi Naito, \\ Satomi Yamada, Kentaro Hozumi, Yamato Kikkawa, and Motoyoshi Nomizu \\ School of Pharmacy, Tokyo University of Pharmacy and Life Sciences, Hachioji, 1920392, Japan
}

\section{Introduction}

It is known that the process of amyloid fibril formation, including amyloid $\beta(A \beta)$ and islet amyloid polypeptide, is generally associated with diseases, such as Alzheimer's disease, type II diabetes, and prion diseases. However, many proteins form amyloid-like fibrils but do not associate with amyloidrelated diseases. Amyloid fibrils are elongated, insoluble structures of 7-10 nm in diameter found in extracellular plaques. Structural studies have shown that mature amyloid fibrils involve the alignment of short peptide segments, usually between 6 and 12 residues in length, from many protein monomers. Together, these aligned polypeptide stretches form the characteristic core structure of the amyloid fibril, the cross $\beta$ sheet, in which $\beta$ strands run perpendicular to the main axis of the fibril. Amyloid fibrils possess a characteristic UV absorbing spectrum at the peak of $540 \mathrm{~nm}$ with Congo red and a characteristic X-ray diffraction pattern. Thus, specific pattern of molecular interactions, rather than nonspecific hydrophobic interactions, lead to ordered structures. Nevertheless, common structural elements responsible for such organized structures have not been identified and the mechanism of amyloid fibril formation is unclear.

Previously, we screened the synthetic peptide library derived from the laminin-111 sequence, and identified five amyloidgenic peptides, A119 (LSNIDYILIKAS, mouse laminin $\alpha 1$ chain, 1321-1332), A208 (AASIKVAVSADR, mouse laminin $\alpha 1$ chain, 2097-2108), AG97 (SAKVDAIGLEIV, mouse laminin $\alpha 1$ chain, 2942-2953), B133 (DISTKYFQMSLE, mouse laminin $\beta 1$ chain, 1367-1378), and B160 (VILQQSAADIAR, mouse laminin $\beta 1$ chain, 1607-1618) [1]. Laminins, major components of basement membranes, regulate multiple biological functions, including cell adhesion, migration, differentiation, neurite outgrowth, wound healing, angiogenesis, and tumor progression. Laminins are a family of heterotrimeric glycoproteins that consist of $\alpha, \beta$, and $\gamma$ chains. So far, five $\alpha$, three $\beta$, and three $\gamma$ chains have been identified and at least 16 laminin isoforms have been reported that are formed by various combinations of each chains. Here, we focused on the AG97 and B160 peptide, which exhibits amyloid-like fibril formation, heparin-dependent cell attachment activity, and neurite outgrowth activity. Our approach was to investigate the essential residues for fibril formation and cell behavior using alanine-substituted peptides.

\section{Results and Discussion}

We prepared two sets of alanine-substituted peptides for AG97 and B160 (Table 1). First, we evaluated amyloid fibril formability of those peptides using Congo red staining. Congo red is a red pigment, which has high affinity for amyloid fibrils. When Congo red binds to amyloid fibrils, the absorption peak shifts from $490 \mathrm{~nm}$ to $540 \mathrm{~nm}$. The absorption peak of Congo red was shifted in AG97 and B160. The absorption peak of Congo red was not shifted in AG97I11A, V12A, B160V1A, I2A, L3A, Q4A, Q5A, and I10A. The $\mathrm{Ile}^{11}$ and $\mathrm{Val}^{12}$ residues of AG97, the $\mathrm{Val}^{1}, \mathrm{Ile}^{2}, \mathrm{Leu}^{3}, \mathrm{Gln}^{4}, \mathrm{Gln}^{5}$, and $\mathrm{Ile}^{10}$ residues of B160 are suggested to be critical for amyloid fibril formation. Next, we measured circular dichroism (CD) spectra of the Ala substituted peptides of AG97 and B160. The CD spectrum of AG97 showed typical $\beta$ sheet structure. The CD spectra of AG97D5A, I11A and V12A showed $\alpha$ helix structure, and those of AG97V4A, I7A and L9A showed random coil structure. The CD spectrum of B160 showed mixed structure ( $\alpha$ helix and $\beta$ sheet). The CD spectra of B160V1A, I2A, Q4A, Q5A and I10A showed $\alpha$ helix structure, and B160S6A, D9A and R12A showed $\beta$ sheet structure. Furthermore, we examined the amyloid-like fibrils of AG97 and B160 with a transmission electron micrograph. AG97S1A, K3A, D5A, G8A, and E10A lost the amyloid-like fibril forming ability of AG97, and B160V1A, I2A, L3A, Q4A, Q5A, and I10A lost that of B160. We hypothesize that the amyloid-like fibril formation needs 
two steps; $1^{\text {st }}$ step, $\beta$ sheet structure formation, $2^{\text {nd }}$ step, aggregation. The results suggested that important residues are different by each step.

Table 1. Synthetic peptides derived from the AG97 and B160 peptide.

\begin{tabular}{|c|c|c|c|}
\hline Peptide & Sequence & Peptide & Sequence \\
\hline AG97 & SAKVDAIGLEIV & B160 & VILQQSAADIAR \\
\hline AG97S1A & AAKVDAIGLEIV & B160V1A & $\underline{\text { AILQQSAADIAR }}$ \\
\hline AG97K3A & SAAEVDAIGLEIV & B160I2A & VÁLQQSAADIAR \\
\hline AG97V4A & SAKADAIGLEIV & B160L3A & VIAQQQSAADIAR \\
\hline AG97D5A & SAKVㅁAIGLEIV & B160Q4A & VILAQQSAADIAR \\
\hline AG97I7A & SAKVDAAGLEIV & B160Q5A & VILQA्ASAADIAR \\
\hline AG97G8A & SAKVDAIA-ALIV & B160S6A & VILQQㄹAAADIAR \\
\hline AG97L9A & SAKVDAIGAEEIV & B160D9A & VILQQSAA_AIAR \\
\hline AG97E10A & SAKVDAIGLAIIV & B160I10A & VILQQSAAD吕R \\
\hline AG97I11A & SAKVDAIGLEA V & B160R12A & VILQQSAADIA \\
\hline AG97V12A & SAKVDAIGLEIA & & \\
\hline
\end{tabular}

AG97 and B160 are reported that these peptides showed cell attachment activity to human fibrosarcoma HT1080 cells [1]. These peptide promoted cell attachment to human dermal fibroblasts (HDFs). AG97K3A, I11A and V12A peptides lost, the AG97V4A and G8A peptides weakened the HDF attachment activity of AG97, and The B160I2A and R12A peptides lost, and the B160L3A and Q4A peptides weakened that of B160. The $\mathrm{Lys}^{3}$, $\mathrm{Ile}^{11}$ and $\mathrm{Arg}^{12}$ residue were critical, $\mathrm{Val}^{4}$ and Gly were important residues for HDF attachment activity of AG97. The Ile ${ }^{2}$ and $\mathrm{Arg}^{12}$ were critical, $\mathrm{Leu}^{3}$ and $\mathrm{Gln}^{4}$ were important residues for HDF attachment activity of B160. The peptides which form amyloid fibrils showed HDF attachment activity. The AG97 and B160 peptides are reported to bind heparin sulfate proteoglycans, which have negative charge on surface [1]. The results showed basic amino acid (Lys and Arg) residues, which had positive charge, were critical for HDF attachment activity.

We examined neurite outgrowth activity with rat pheochromocytoma PC12 cells. AG97K3A, V4A, I11A, and V12A peptides lost the neurite outgrowth activity of AG97. The $\mathrm{Lys}^{3}, \mathrm{Val}^{4}, \mathrm{Ile}^{11}$, and $\mathrm{Val}^{12}$ residue were critical residues for the neurite outgrowth activity of AG97. B160I2A, L3A, Q4A, and R12A peptides lost the neurite outgrowth activity of $\mathrm{B} 160$. The $\mathrm{Ile}^{2}, \mathrm{Leu}^{3}, \mathrm{Gln}^{4}$, and $\mathrm{Arg}^{12} \mathrm{residue}^{2}$ were critical residues for the neurite outgrowth activity of B160. The peptides which form amyloid fibrils showed neurite outgrowth activity. The result suggested that the basic amino acids also play important role for neurite outgrowth activity.

The results suggested that the biological activities (HDF attachment and neurite outgrowth) were closely related with amyloid-like fibril formation. Furthermore, the results indicated that the Ile residue was critical for fibril formation. These findings were useful to elucidate the mechanism of amyloidlike fibril formation.

\section{Acknowledgments}

This work was supported by JSPS KAKENHI Grant Number 26870613.

\section{References}

1. Kasai, S., et al. Biochemistry 46, 3966-3974 (2007), http://dx.doi.org/10.1021/bi062097t

2. Katagiri, F., et al. Biochemistry 51, 8218-8225 (2012), http://dx.doi.org/10.1021/bi300822d

3. Yamada, M., et al. FEBS Lett 530, 48-52 (2002), http://dx.doi.org/10.1016/S0014-5793(02)03393-8

4. Katagiri, F., et al. Biochemistry 49, 5909-5918 (2010), http://dx.doi.org/10.1021/bi100748s 